\title{
¿Qué opinan adolescentes y jóvenes sobre el consumo de drogas recreativas y las conductas sexuales de riesgo?
}

\author{
Ainhoa Rodríguez García de Cortázar*; Mariano Hernán García**; Andrés Cabrera león**; \\ José María García Calleja****; Nuria Romo Avilés*****. \\ * Especialista de Proyectos del Observatorio de la Infancia en Andalucía. \\ * * Director del Observatorio de la Infancia en Andalucía. \\ *** Coordinador de estudios y proyectos del Observatorio de la Infancia en Andalucía. \\ **** Departamento de Medición y Sistemas de Información (EIP/MHI unit) de la Organización Mundial para la Salud (WHO), Ginebra. \\ ***** Profesora Asociada. Departamento de Antropología Social. Universidad de Granada. \\ Enviar correspondencia a: \\ Ainhoa Rodríguez García de Cortázar. Observatorio de la Infancia en Andalucía. C/ Paseo de los Coches s/n (Alhambra), 18009 Granada. \\ Tfn: 958029769/60. Fax: 958029775. ainhoa.rodriguez@juntadeandalucia.es
}

\section{RESUMEN}

El objetivo de este artículo es analizar las opiniones de adolescentes y jóvenes, de población gitana y no gitana, sobre la relación entre el consumo de drogas recreativas y las prácticas sexuales que incrementan el riesgo de infección por VIH. Para ello se ha desarrollado un estudio transversal descriptivo que emplea una metodología cualitativa. Se realizaron 14 grupos focales en los que participaron 98 adolescentes y jóvenes y 7 entrevistas semiestructuradas a jóvenes usuarios de drogas recreativas. Los resultados fueron triangulados.

En el análisis aparecen dos grandes líneas discursivas. La primera defiende que el consumo moderado de alcohol facilita el encuentro sexual, pero no implica la asunción de riesgos. Sin embargo, el policonsumo o consumo elevado de drogas recreativas se relaciona con la despreocupación por los riesgos sexuales, y en varones con pérdida de sensibilidad sexual que justifica el no uso de preservativos. La segunda argumenta otros motivos para no utilizar preservativos, entre ellos la falta de disponibilidad de los mismos, la confianza en la pareja sexual, una concepción del deseo como algo incontrolable, el enamoramiento, el estado de ánimo o la autoestima.

De los resultados se derivan recomendaciones para prevenir la transmisión sexual del VIH, como distribuir preservativos en espacios donde se consume alcohol y otras drogas, publicitar su uso y el de otras barreras en prácticas bucogenitales, trabajar en la optimización del placer con varones, convertir en objeto de campañas a las parejas estables y formar a mujeres adolescentes en habilidades sociales para negociar el uso del preservativo.

Palabras clave: adolescente, uso drogas. conducta sexual de riesgo, uso preservativos, VIH, metodología cualitativa.

\section{ABSTRACT}

The objective of this article is to analyse the opinions of adolescents and young people, from gypsy and non-gypsy populations, on the relationship between recreational drug use and sexual practices that increase the risk of HIV infection.

A descriptive qualitative research was undertaken. 14 focus groups were conducted with 98 adolescents and young people, and 7 semi-structured interviews with young recreational drug users. Both sort of results were triangulated.

Two major discursive lines emerge in the analysis. The first one defends the notion that moderate consumption of alcohol facilitates the sexual encounter, but it does not imply risky behaviours. However, polydruguse or an elevated use of recreational drugs is related to a lack of concern for sexual risks, and in men with the loss of sexual sensitivity that sometimes justifies not using a condom. The second line argues other reasons for the non-use of condoms, such as their lack of availability, confidence in one's sexual partner, a concept of desire as something uncontrollable, infatuation and the state of mind or self-esteem.

Some recommendations to prevent sexual transmission of HIV are derived from the results, such as the distribution of condoms in places where alcohol and other drugs are consumed, publicising the use of condoms and other latex barriers for oral genital sexual practices, working with young males on the optimisation of pleasure, designing preventive interventions targeting stable partners, and training adolescent girls in the social skills needed to negotiate the use of condoms.

Keywords: adolescent, use of drugs, risk sexual behaviour, HIV, condoms, qualitative research. 


\section{INTRODUCCIÓN}

$\mathrm{L}$ a Organización Mundial para la Salud (OMS) define drogas o sustancias psicoactivas como aquelas cuyo consumo afecta al proceso mental ${ }^{1}$. En los últimos años se viene observando un incremento del consumo de alcohol y drogas ilegales, excepto heroína, entre la juventud europea ${ }^{2}$. Parece haberse producido un cambio en la representación social del uso de sustancias psicoactivas en los jóvenes; según el ambiente y la moda, se toman unas drogas $u$ otras $^{3,4}$. Asimismo, se observa que lo más frecuente es el policonsumo ${ }^{2}$, donde el alcohol actúa como catalizador del uso del resto de sustancias ${ }^{5}$. A la par, las comunicaciones y el movimiento de poblaciones facilitan que la disponibilidad de distintas drogas atraviese barreras culturales y geográficas. El uso de drogas en las sociedades actuales constituye un fenómeno complejo, por lo que su estudio exige tener en cuenta tanto las propiedades farmacológicas de cada sustancia como las expectativas asociadas a las mismas, las pautas de consumo, las condiciones de vida y las normas sociales sobre cada sustancia ${ }^{6}$.

Por otro lado, investigaciones sobre el consumo de drogas en la juventud muestran éste como un comportamiento de riesgo que puede estar relacionado con otras conductas "arriesgadas"7,8. El riesgo es una construcción social, histórica y culturalmente variable. Lo que se considera riesgo desde determinadas posiciones sociales no es visto como tal desde otros sectores $^{9}$. La edad, la clase social o las condiciones de vida determinan la escala de valores de las personas y el nivel de riesgos con el que coexisten o que están dispuestas a asumir. Por ejemplo, la percepción y la acción frente a situaciones de riesgo se relaciona con los roles de género definidos socialmente. Tradicionalmente, las mujeres no alcanzan el mismo estatus positivo al relacionarse con conductas de riesgo que los varones ${ }^{7}, 10$.

Además, organismos internacionales estiman que alrededor de la mitad de las nuevas infecciones por $\mathrm{VIH}$ se producen en menores de 25 años ${ }^{11}, 12$. El Programa Conjunto de las Naciones Unidas para el Sida (ONUSIDA) en su informe global de 2006 calcula que un $40 \%$ de las nuevas infecciones se producen en personas de 15 a 24 años de edad ${ }^{13}$. El contacto heterosexual aparece en algunos países de Europa Occidental como causa predominante de las nuevas infecciones ${ }^{14}$. En España, se viene observando un aumento del porcentaje de casos de SIDA por transmisión sexual, porcentaje más elevado entre las mujeres, y una mayor frecuencia relativa de casos de VIH en las jóvenes que en las adultas ${ }^{15}$. No obstante, el porcentaje de uso regular o constante de preservati- vos con parejas ocasionales sigue siendo menor entre las personas adultas que en las jóvenes ${ }^{16}$.

Enfrentar el "riesgo" que supone mantener relaciones sexuales sin protección frente al VIH o, desde otro punto de vista, utilizar preservativos depende de una multiplicidad de factores ${ }^{17}$. Cuestiones como la salud mental ${ }^{18}$, el estatus socioeconómico ${ }^{19}$, el capital socia ${ }^{20}$ y otros elementos contextuales ${ }^{21}$ pueden influir tanto en las prácticas sexuales que incrementan el riesgo de infección por VIH como en el uso de ciertas drogas. En este sentido, ONUSIDA y OMS plantean una serie de estrategias múltiples e integradas para lograr cambios en los contextos estructural, comunitario e interpersonal, con el fin de intervenir en el binomio consumo de sustancias psicoactivas y conductas sexuales de riesgo 22 .

Por otro lado, la relación entre el consumo de drogas recreativas y las prácticas sexuales que incrementan el riesgo de infección por VIH puede presentar particularidades en adolescentes y jóvenes de población gitana en riesgo de exclusión social ${ }^{23}$. Se trata de una población demográficamente joven ${ }^{24}$ y vulnerable al abuso de drogas, lo que está dañando la imagen de la comunidad gitana, y está suponiendo una dificultad más a su promoción e integración social25. Asimismo, instituciones como el Observatorio Europeo del Racismo y la Xenofobia (EUMC) hacen hincapié en la dificultad que tienen para acceder a información sobre enfermedades de transmisión sexual mujeres adolescentes y jóvenes de familias o comunidades con tradiciones que asocian su virginidad con el honor familiar ${ }^{26,27}$.

La relación entre el consumo de drogas y las prácticas sexuales que incrementan el riesgo de infección por VIH en adolescentes y jóvenes ha sido estudiada anteriormente, si bien los resultados no son concluyentes ${ }^{28}$. Hay investigaciones que asocian el consumo de alcohol ${ }^{29},{ }^{30} \mathrm{u}$ otras sustancias psicoactivas ${ }^{31}$ con el no uso de preservativos ${ }^{32,33}$, pero también las hay que no encuentran tal asociación ${ }^{34,35,36,37}$. La relación no es simple. Diversos autores han destacado la importancia de las expectativas individuales sobre los efectos del alcohol en el comportamiento sexual38, 39 . Algunos defienden que la psicoactividad de una sustancia no establece la desinhibición o el control del comportamiento, simplemente facilita una conciencia alterada sobre cultura, circunstancias y personalidad ${ }^{39}$.

Estamos ante un asunto complejo, por ello el objetivo de este trabajo ha sido aproximarse al fenómeno y analizar opiniones de adolescentes y jóvenes, de población gitana y no gitana, sobre su contexto sociocultural y la visión que tienen de la relación entre el 
consumo de drogas y las prácticas sexuales que incrementan el riesgo de infección por VIH. Y por otro lado, es también propósito de este trabajo recoger recomendaciones útiles para diseñar acciones preventivas de la transmisión sexual del VIH y de las drogodependencias con estas poblaciones.

\section{MÉTODOS}

Se ha desarrollado un estudio descriptivo y transversal, empleado una metodología cualitativa. Ésta facilita la comprensión de los diferentes sentidos que dan adolescentes y jóvenes al consumo de drogas, desde sus propias perspectivas. A la vez, permite el conocimiento de experiencias vitales y el análisis de la influencia de los contextos sociales, económicos y culturales en los significados asociados al consumo de drogas y a las conductas sexuales que incrementan el riesgo de infección por $\mathrm{VIH}$.

El trabajo de campo se realizó entre los meses de octubre de 2003 y mayo de 2004 en Granada, una provincia del sur de España.

Se ha utilizado un diseño muestral intencional, seleccionando a las personas participantes según perfiles previamente establecidos. Para la captación de adolescentes y jóvenes, así como de informantes clave, se ha recurrido al método bola de nieve ${ }^{40}$ así como a la colaboración de orientadores en centros educativos o Escuelas Taller y de asociaciones juveniles que trabajan con adolescentes y jóvenes en riesgo de exclusión social pertenecientes mayoritariamente a la comunidad gitana.

La información se ha obtenido por medio de 14 grupos focales, en los que participaron un total de 98 adolescentes y jóvenes, 7 entrevistas semiestructuradas a jóvenes con perfiles específicos y un grupo nominal con menores de 18 años en riesgo de exclusión social de la comunidad gitana.

Los criterios de inclusión en grupos focales y entrevistas han sido tener entre 16 y 29 años de edad, consumir drogas recreativas y haber mantenido relaciones sexuales*
Un grupo piloto permitió realizar las pruebas y modificaciones pertinentes al diseño inicial, mejorar el guión y cerrar los criterios de segmentación.

A las personas participantes en los grupos focales se les entregó un cuestionario anónimo para recoger datos sociodemográficos, hábitos de consumo de cada una de las distintas drogas recreativas, referentes de consumo de las mismas en el entorno, tipos de relaciones sexuales, nivel de uso de preservativos $u$ otras barreras y referentes de conductas sexuales de riesgo en el entorno.

Además de las diferencias étnicas, de edad y de hábitos de consumo de drogas, se ha procurado acceder a adolescentes y jóvenes de diferentes clases sociales, controlando la procedencia urbana (zona centro / barrios en riesgo de exclusión social) y el nivel de instrucción. La situación laboral más frecuente ha sido el desempleo o empleo de baja cualificación, habitual para las edades estudiadas.

Finalmente la muestra se compuso de 54 mujeres y 44 varones de entre 16 y 29 años de edad, el 38\% de estas personas eran gitanas. La gran mayoría eran usuarias habituales de alcohol y/o cannabis, y alrededor del 36\% consumían habitualmente drogas de síntesis u otras drogas además de tabaco, alcohol y cannabis. El 86\% de las y los participantes explicitó el tipo de relaciones sexuales que mantiene; declarando el $37 \%$ relaciones ocasionales, el $24 \%$ pareja estable y relaciones ocasionales simultáneamente, el $23 \%$ pareja estable y el $16 \%$ una sucesión de parejas estables (monogamia seriada).

Las moderaciones de los grupos focales fueron dirigidas por tres personas sociólogas, una antropóloga y dos animadoras socioculturales debidamente entrenadas; profesionales de etnia gitana para los grupos de gitanos, mujeres para los grupos mixtos y de mujeres y varones para los grupos de varones (excepto uno).

Las entrevistas semiestructuradas a jóvenes con perfiles específicos han permitido recoger declaraciones más íntimas y alejadas de los discursos socialmente "correctos" o deseables. Igualmente facilitaron una visión procesual de las trayectorias de las personas entrevistadas, en relación al consumo de sustancias psicoactivas y a los cambios en sus comportamientos sexuales. Además las entrevistas sirvieron para facilitar la triangulación de técnicas, enriqueciendo la información de los grupos focales.

\footnotetext{
* Debido a cuestiones de origen cultural, este último requisito no se hizo explícito para las gitanas menores de edad solteras o sin pareja reconocida.
} 
Tabla 1. Perfil de los grupos focales o triangulares realizados

\begin{tabular}{|c|c|c|c|c|c|}
\hline $\begin{array}{l}\text { Código } \\
\text { grupo }\end{array}$ & 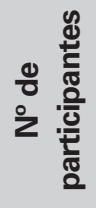 & Perfil & 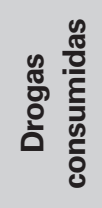 & $\begin{array}{l}\text { Formación } \\
\text { académica }\end{array}$ & Situación laboral \\
\hline GF01 & 9 & $\begin{array}{c}\text { Mujeres y varones de } 1 \\
8 \text { a } 29 \text { años }\end{array}$ & a & Universitaria & $\begin{array}{c}\text { No trabaja y trabajo } \\
\text { temporal }\end{array}$ \\
\hline GF02 & 6 & $\begin{array}{l}\text { Mujeres y varones de } \\
25 \text { a } 29 \text { años }\end{array}$ & $b$ & No universitaria & Trabajo no cualificado \\
\hline GF03 & 7 & $\begin{array}{l}\text { Mujeres y varones de } \\
\qquad 18 \text { a } 24 \text { años }\end{array}$ & $b$ & $\begin{array}{c}\text { Universidad y estudios } \\
\text { artísticos }\end{array}$ & Trabajo no cualificado \\
\hline GF04 & 7 & $\begin{array}{l}\text { Mujeres de } \\
18 \text { a } 24 \text { años }\end{array}$ & a & $\begin{array}{l}\text { Universitarias y } \\
\text { estudiantes de FP }\end{array}$ & $\begin{array}{c}\text { No trabaja y trabajo } \\
\text { temporal }\end{array}$ \\
\hline GF05 & 8 & $\begin{array}{l}\text { Mujeres de } \\
16 \text { y } 17 \text { años }\end{array}$ & a & $\begin{array}{c}\text { Estudiantes de ESO, } \\
\text { Bachillerato y Programas } \\
\text { de Garantía Social }\end{array}$ & No trabaja \\
\hline GF06 & 6 & $\begin{array}{l}\text { Varones de } \\
16 \text { y } 17 \text { años }\end{array}$ & a & ESO y Bachillerato & Mayoría no trabaja \\
\hline GF07 & 7 & $\begin{array}{l}\text { Mujeres gitanas de } \\
18 \text { a } 24 \text { años }\end{array}$ & a & Escuela Taller & Trabajo no cualificado \\
\hline GF08 & 5 & $\begin{array}{l}\text { Varones de } \\
18 \text { a } 24 \text { años }\end{array}$ & $b$ & Escuela Taller & Albañilería \\
\hline GT09 & 3 & $\begin{array}{l}\text { Hombres de } \\
18 \text { a } 24 \text { años }\end{array}$ & a & Escuela Taller & $\begin{array}{c}\text { Electricidad, carpintería, } \\
\text { fontanería }\end{array}$ \\
\hline GF10 & 7 & $\begin{array}{l}\text { Mujeres de } \\
18 \text { a } 24 \text { años }\end{array}$ & $b$ & Bachillerato y universitaria & No trabaja \\
\hline GF11 & 10 & $\begin{array}{l}\text { Varones gitanos de } \\
\qquad 18 \text { a } 24 \text { años }\end{array}$ & b & $\begin{array}{c}\text { Primaria y primaria } \\
\text { inconclusa }\end{array}$ & Albañiles y jornaleros \\
\hline GF12 & 10 & $\begin{array}{l}\text { Varones gitanos de } \\
16 \text { y } 17 \text { años }\end{array}$ & a & ESO & No trabaja \\
\hline GF13 & 10 & $\begin{array}{l}\text { Mujeres gitanas de } \\
16 \text { y } 17 \text { años }\end{array}$ & a & Escuela Taller & $\begin{array}{c}\text { Electricidad, carpintería, } \\
\text { fontanería }\end{array}$ \\
\hline GT14 & 3 & $\begin{array}{l}\text { Mujeres lesbianas de } \\
18 \text { a } 24 \text { años }\end{array}$ & a & Universitaria & $\begin{array}{l}\text { Supermercado, } \\
\text { hostelería }\end{array}$ \\
\hline
\end{tabular}

a. Tabaco, alcohol y/o cannabis.

b. Otras drogas además de alcohol y/o cannabis; éxtasis, cocaína, speed, ketamina, LSD, hongos alucinógenos, opio, heroína inhalada.

Los grupos focales y las entrevistas se transcribieron literalmente. Las dimensiones y subdimensiones de análisis fueron trianguladas por tres investigadores. A continuación se presenta una tabla con las categorías creadas para el análisis.
Se realizó un análisis de contenido con el programa Atlas.ti $4.2^{41}$ y una triangulación de los resultados, mediante el análisis independiente y contrastado de dos investigadores. 
Tabla 2. Perfil de las entrevistas realizadas

\begin{tabular}{|l|l|}
\hline E01 & Joven camarero en discotecas, $\mathrm{VIH}(+)$ por transmisión sexual \\
\hline E02 & Joven camarero en bar nocturno, consumidor habitual de alcohol, cannabis y ocasional de otras drogas \\
\hline E03 & Joven no consumidor de drogas \\
\hline E04 & Joven usuario habitual de alcohol y drogas de síntesis \\
\hline E05 & Joven usuaria habitual de alcohol y drogas de síntesis \\
\hline E06 & Joven homosexual policonsumidor \\
\hline E07 & Joven homosexual consumo moderado de alcohol y otras drogas \\
\hline
\end{tabular}

Tabla 3. Dimensiones y subdimensiones de análisis.

\begin{tabular}{|c|c|c|}
\hline Dimensiones & \multicolumn{2}{|c|}{ Subdimensiones } \\
\hline \multirow{12}{*}{ VIH } & Información sobre VIH & \\
\hline & Percepción del VIH & \\
\hline & Relación sexualidad - VIH & \\
\hline & Prácticas de riesgo & \\
\hline & Prácticas seguras & \\
\hline & Métodos de prevención: & Preservativos \\
\hline & \multirow{3}{*}{ Prevención VIH: } & Intervenciones \\
\hline & & Obstáculos a la prevención \\
\hline & & Información sobre prevención \\
\hline & Enfermedades de Transmisión Sexual & \\
\hline & \multirow{2}{*}{ Diferencias de género respecto al VIH: } & Roles tradicionales/nuevos \\
\hline & & Relaciones de poder \\
\hline \multirow{17}{*}{ Drogas } & Salud y drogas & \\
\hline & Percepción del sexo bajo drogas & \\
\hline & Alcohol & \\
\hline & Cannabis & \\
\hline & Éxtasis (MDMA) & \\
\hline & Cocaína, Speed & \\
\hline & LSD, Hongos alucinógenos & \\
\hline & Psicofármacos, Ketamina, etc. & \\
\hline & Opiáceos & \\
\hline & Tabaco & \\
\hline & Policonsumo & \\
\hline & Adicción & \\
\hline & \multirow{3}{*}{ Prevención drogas: } & Intervenciones \\
\hline & & Obstáculos a la prevención \\
\hline & & Información sobre prevención \\
\hline & \multirow{2}{*}{ Género y drogas: } & Roles tradicionales/nuevos \\
\hline & & Relaciones de poder \\
\hline \multirow{11}{*}{ Relaciones sexuales } & Modelos sexuales & \\
\hline & Espacios y tiempos & \\
\hline & Problemas sexuales & \\
\hline & Tipo de parejas sexuales & \\
\hline & Educación sexual & Proposiciones sexuales \\
\hline & Comunicación / negociación & \\
\hline & Riesgos sexuales & \\
\hline & Sexo oral / anal & \\
\hline & Otras prácticas sexuales & \\
\hline & \multirow{2}{*}{ Relaciones sexuales y género: } & Roles tradicionales/nuevos \\
\hline & & Relaciones de poder \\
\hline
\end{tabular}




\begin{tabular}{|c|c|c|}
\hline \multirow{11}{*}{ Riesgo, sexo y drogas } & Relación drogas / sexo inseguro & \\
\hline & Relación drogas / sexo seguro & \\
\hline & \multirow{7}{*}{ Otros factores de riesgo: } & Disponibilidad de preservativo \\
\hline & & Confianza en la pareja \\
\hline & & Enamoramiento \\
\hline & & Sexo $=$ droga \\
\hline & & Deseo como algo irrefrenable \\
\hline & & Estado de ánimo \\
\hline & & Ideología \\
\hline & Normas sociales sobre sexo y drogas & \\
\hline & Género, riesgos, sexo y drogas & \\
\hline \multirow{3}{*}{ Adolescencia y juventud } & Valores & \\
\hline & Estereotipos & \\
\hline & Programas & \\
\hline
\end{tabular}

Los resultados fueron presentados a la población objeto de estudio en una sesión de devolución. El hecho de incluir esta devolución de la información a los sujetos de estudio se justifica desde una perspectiva ética y de eficacia social de las posibles intervenciones preventivas que de los resultados se deriven. Se reconoce de manera práctica la calidad de sujeto al objeto de estudio, y se le permite participar en la elaboración final de la investigación. Para ello se realizaron una exposición de viñetas con extractos literales de los grupos focales, ejemplificando los principales discursos recogidos, y un grupo nominal con la intención de recoger recomendaciones para la prevención de la transmisión sexual del VIH. En la técnica participaron 14 menores de 18 años, de población gitana en riesgo de exclusión. La pregunta motor fue; “¿qué se os ocurre que se podría hacer para motivar a los y las jóvenes a que utilicen preservativos?".

La participación en la investigación ha sido voluntaria, si bien como muestra de agradecimiento por el tiempo destinado a la investigación se dio a las personas participantes un vale canjeable por un disco o libro. En todo momento se ha garantizado el anonimato de las personas participantes, y sus datos personales se han guardado de forma disociada en ficheros de seguridad. Por último, se les ha suministrado toda la información solicitada sobre la investigación (salvo la protegida por razones de seguridad) y sobre cuestiones relacionadas con la misma, como prevención de prácticas sexuales que incrementan el riesgo de infección por VIH.

\section{RESULTADOS}

\section{Contexto sociocultural en el que se llevan a cabo las prácticas estudiadas}

Los hábitos de consumo de sustancias psicoactivas y las relaciones sexuales que establecen adoles- centes y jóvenes se desarrollan en un contexto social y cultural determinado. Los espacios donde se suelen iniciar los encuentros sexuales, los modelos sexuales dominantes, las normas sociales y la educación sexual, lo que se considera riesgo y lo que se considera práctica segura en su entorno o las dificultades que expresan algunas mujeres para negociar el uso de preservativos, influyen en sus relaciones y prácticas sexuales. Por ello, se han tenido en cuenta las percepciones que de los mismos tienen las y los adolescentes y jóvenes participantes, con la intención de contextualizar los resultados de la investigación.

Las personas consultadas relacionan los espacios de ocio nocturno y los contextos de fin de semana, donde se hace uso de drogas recreativas, con la posibilidad de iniciar un encuentro sexual. Este encuentro sexual se llevará a cabo en diversos lugares, según las posibilidades de la pareja -casa propia, de los padres, coche, calle, el servicio de un bar, etc.- lo que puede influir en la tranquilidad con la que se realiza el acto sexual concreto.

"Varón: Fin de semana, entonces se tienen relaciones bajo efectos del alcohol u otras drogas, porque los colegas míos que follaban sin condón, siguen follando sin condón cuando están pedos y cuando no, lo que pasa es que cuando van pedos suele ser más que cuando no van, por la situación social del ligoteo y tal, el fin de semana es cuando sale todo el mundo"

Grupo 2. Varones y mujeres de 25 a 29 años consumidores de otras drogas además de alcohol y/o cannabis.

Además, opinan que desde los medios de comunicación se incita al consumo, a los excesos, al disfrute del ahora, de la novedad y de las sensaciones fuertes. Sin embargo, también dicen recibir mandatos sociales contrarios, prohibiendo aquello que resulta placentero, especialmente en el tiempo productivo, de lunes a viernes. Esta aparente contradicción de normas 
sociales, se traduce en prácticas de consumo elevado de drogas recreativas y en intentos de relacionarse sexualmente.

"yo creo que la sociedad te inculca eso, el disfrute del hoy y que lo que venga después pues... que sea lo que dios quiera. (...) Si la sociedad misma fomenta que la gente juegue, practique sexo como descosidos y que tome drogas (...) Primero, te dicen que practicar sexo tiene un riesgo muy grande de enfermedades; luego los médicos te dicen que el sexo es muy bueno para la salud, eso rejuvenece."

Grupo 11. Varones gitanos de 18 a 24 años consumidores de otras drogas además de alcohol y/o cannabis.

Por otro lado, salvo en el grupo de jóvenes lesbianas, se visibiliza la asunción por ambos sexos de un modelo sexual dominante donde la penetración juega un papel central. En este sentido, las propias participantes de varios grupos focales han definido estos modelos como "masculinizados", centrados en la sexualidad del varón. Aparece así la idea de que evitar riesgos supone evitar la penetración.

"vamos, que el sexo yo creo que está construido totalmente para ellos. El sexo entendido como lo entendemos hoy en día; la penetración"

Grupo 10. Mujeres de 18 a 24 años consumidoras otras drogas además de alcohol y/o cannabis.

"a lo mejor puede haber juegos que puedes hacer con tu novio, que digas; 'bueno pues no te hace falta penetración', pero... Yo creo que no es lo mismo"

Grupo 5. Mujeres de 16 y 17 años consumidoras de alcohol y/o cannabis.

Asimismo, una buena parte de las y los jóvenes consultados consideran pobre y represiva la educación sexual recibida, lo que, en su opinión, influye en los miedos o habilidades que tienen a la hora de proponer relaciones sexuales. De este modo, mujeres y varones participantes reconocen que utilizan el alcohol y otras drogas esperando un efecto desinhibidor.

"cómo entrarle a una persona, cómo simplemente ser sincero contigo mismo y con la gente, eso no te lo enseñan en el cole. Y la gente tiene un montón de miedos y parece que no sabe relacionarse sin droga"

Entrevista 4. Joven usuario de alcohol y drogas sintéticas.

Mujeres menores de 25 años y sobre todo adolescentes señalan dificultades para comunicar sus deseos y para negociar el uso del preservativo. "porque esa persona nunca, que ya me lo había dicho que no follaba con condón. Y claro, yo también me quedé ahí muy parada, ¿no? Cuando tienes claro que a la otra persona no le va a molar nada y la ves muy lanzada pues... Igual si no tienes confianza te cuesta el decirle en un momento dado -ponte un condón- y a lo mejor la primera vez no supe reaccionar."

Grupo 4. Mujeres de 18 a 24 años consumidoras de alcohol y/o cannabis.

Algunas entrevistadas de mayor edad hablan sobre un proceso de aprendizaje y "empoderamiento" necesario para superar estas dificultades.

"primero te drogas de una manera, follas de una manera y luego te das cuenta de que así no te cuadra e intentas hacerlo mejor o por lo menos que te sea más satisfactorio. (...) Y menos arriesgado también"

Grupo 2. Varones y mujeres de 25 a 29 años consumidores de otras drogas además de alcohol y/o cannabis.

Las personas participantes en la investigación entienden que son riesgos sexuales, bajo los efectos de drogas recreativas, el embarazo y las infecciones de transmisión sexual, incluido el VIH/SIDA. Otro riesgo explicitado exclusivamente por mujeres son las relaciones sexuales no deseadas.

"¿Riesgos?, pues que lo hagas con una persona que no quieras"

Grupo 5. Mujeres de 16 y 17 años consumidoras de alcohol y/o cannabis.

Normalmente califican el sexo bucogenital como una práctica "segura", o al menos más segura que la penetración sin preservativo y, salvo un joven, declaran no utilizar para ella ningún tipo de barreras. Prefieren realizar esta práctica sin protección, o no llevarla a cabo, a proponer para ella el uso de preservativo u otras barreras, algo que afirman nada común en su entorno.

"M: En el sexo oral y todo eso, todo ese tipo de enfermedades estoy, me arriesgo a cogerlas, ¿no?, realmente me estoy arriesgando a cogerlas. Drogada y sin drogar"

Grupo 3. Mujeres y varones de 18 a 24 años consumidores de otras drogas además de alcohol y/o cannabis.

En síntesis, las personas adolescentes y jóvenes consultadas opinan que viven en un contexto sociocultural donde las noches del fin de semana se consideran momentos habituales para proponer relaciones sexuales, momentos donde se suelen consumir drogas recreativas, que a su vez se perciben como faci- 
litadoras de la desinhibición sexual. El modelo sexual predominante prima la penetración vaginal, práctica considerada de riesgo si se realiza sin preservativos u otros anticonceptivos, frente a las relaciones bucogenitales, que se suelen practicar sin ningún tipo de barrera protectora.

\section{Conductas sexuales bajo los efectos de drogas recreativas}

Las opiniones recogidas de adolescentes y jóvenes que consumen drogas recreativas pueden agruparse en dos grandes líneas discursivas. La primera la constituyen aquellas afirmaciones que argumentan que existe una relación entre el consumo de drogas recreativas y las prácticas sexuales de riesgo, ya sea positiva o negativa. La segunda linea agrupa aquellos razonamientos que defienden que si no se utilizan preservativos bajo los efectos de drogas recreativas se debe a otras cuestiones, más allá del mero uso de sustancias psicoactivas. Ambas líneas coexisten y conforman puntos de vista que se superponen, y en ocasiones coexisten en el discurso de una misma persona.

En la primera línea discursiva, la población consultada tiene en cuenta dos dimensones o variables que considera fundamentales a la hora de afirmar la existencia de una relación entre el consumo de drogas recreativas y las conductas sexuales de riesgo. Por un lado, la percepción sobre la cantidad consumida, es decir, si se trata de consumo y/o policoncumo moderado, elevado o abusivo de drogas recreativas, y por otro lado, la percepción y expectativas sobre los efectos concretos de cada sustancia.

Las y los consultados consideran que el uso moderado de drogas como el alcohol facilita el establecimiento de los contactos previos a una relación sexual, pero no relacionan este nivel de consumo con la asunción de riesgos sexuales. Sin embargo, opinan que un consumo elevado, y sobre todo un policonsumo de sustancias como éxtasis, alcohol y speed o cocaína, puede llevar a una pérdida del control de la situación que derive en la realización de prácticas sexuales de riesgo.

"Sabes lo que estás haciendo, pero no te fijas en todos los detalles. (...) Sabes a quién te estás tirando, pero no te pones a pensar (...) -tengo que coger el preservativo, tengo que buscar un sitio donde no haya gente, tengo que no sé qué, tengo que no sé cuánto-. No, porque además, es que eso, es que es aburrido, por lo menos para mí"

Grupo 5. Mujeres de 16 y 17 consumidoras de alcohol y/o cannabis.

Por otro lado, las y los consultados consideran que un consumo abusivo de alcohol o un policonsumo excesivo de drogas recreativas impide o dificulta las relaciones sexuales con penetración y, por tanto, disminuye el riesgo de infección por VIH u otras ITS.

"Hombre, pero se supone que si tú vas muy ciego no se te levanta"

Grupo 6. Varones de 16 y 17 consumidores de alcohol y/o cannabis.

Destaca la "conciencia" expresada en la exposición a riesgos sexuales bajo los efectos de drogas recreativas. Así, tras el uso de sustancias como alcohol, éxtasis, speed, cocaína y ketamina dicen haber asumido conscientemente los riesgos derivados de una práctica sexual sin protección. En ocasiones por no tener un preservativo a mano.

“Ya, ya, a mí por ejemplo, cuando estoy borracha y no tengo preservativo, hay un momento que da igual, que si hay bien, y si no hay también."

Grupo 1. Varones y mujeres de 18 a 29 años consumidores de alcohol y cannabis.

La decisión de no relacionarse sexualmente bajo los efectos de un consumo elevado de sustancias psicoactivas es expresada por un sector de las personas consultadas de mayor edad y/o con más experiencia "drogándose".

También es percibida una separación entre "sexo" y "drogas" en ciertas "raves" o ambientes de baile minoritarios. En opinión de una joven, en ellos prima el consumo de sustancias recreativas y está mal visto relacionarse con otras personas con un objetivo sexual.

"Claro, para pasártelo bien y no metes el sexo por medio, porque como que queda feo incluso el ligoteo ese de antes de (...) de 'me gustas', ¿no? 'iQué haces, chico! Yo estoy aquí poniéndome hasta el culo, paso de todo', ¿no?"

Grupo 3. Mujeres y varones de 18 a 24 años consumidores de otras drogas además de alcohol y/o cannabis.

En referencia a la posible influencia en las prácticas sexuales diferenciada en función del tipo de sustancia psicoactiva, declaran que bajo los efectos de alcohol o éxtasis, así como de un policonsumo de speed o cocaína, y éxtasis, además de alcohol y/o cannabis, le han dado menos importancia al uso de preservativos. El disfrute del momento y el placer encontrado en la reducción del control -expectativas asociadas al uso de drogas recreativas- son expresados como facilitadores de las prácticas sexuales de riesgo.

"se me ha ido de las manos sí, pues drogada sí, de pastillas, de speed o de ketamina. He estado 
follando con personas que he dicho, me he arriesgado (...) estás tan a gusto que no quieres pensar en los riesgos"

Grupo 3. Mujeres y varones de 18 a 24 años consumidores de otras drogas además de alcohol y/o cannabis.

Sin embargo, no todas las sustancias se relacionan con la asunción de riesgos sexuales. Por ejemplo, en opinión de usuarios habituales, el consumo elevado de cannabis mezclado con tabaco, sin combinar con otras drogas, no influye en la realización de prácticas sexuales de riesgo. El speed también es percibido por las y los consumidores consultados como una droga que por sí sola no facilita la pérdida de control en este aspecto. Asimismo, las personas consultadas declaran no mantener relaciones sexuales bajo los efectos de LSD u hongos alucinógenos.

Sobre la cocaína está presente la percepción o creencia de que permite "aguantar" más tiempo sin eyacular. Esto a su vez se considera socialmente necesario para hacer disfrutar a la pareja sexual, es decir está asociado al estereotipo por el que se asume que cuánto más dure el acto sexual más satisfactorio es para la mujer. Sin embargo, la pérdida de sensibilidad sexual y las dificultades para alcanzar el orgasmo bajo los efectos de sustancias como alcohol o cocaína, son argumentos que expresan los varones consultados para no utilizar el preservativo, o para quitárselo antes de concluir la penetración sexual. A la reducción del placer percibido bajo los efectos de cocaína se le suma la disminución de sensibilidad derivada del uso de dicha barrera.

"Ya sin condón no te corres (con la cocaína), pues imagínate con condón, que todavía sientes menos"

Grupo 8. Varones de 18 a 24 años consumidores de otras drogas además de alcohol y/o cannabis.

Por último, sustancias como el opio o la heroína no inyectada se asocian por las y los consultados usuarios esporádicos de las mismas a dificultades para mantener la erección. Por ello consideran que los riesgos derivados de una penetración vaginal $u$ anal sin preservativo son menores que con otras sustancias.

"a mí para follar siempre me ha gustado mucho el opio (...) lo que pasa que claro, que los chicos no se empalman; te tienes que olvidar un poco de la penetración, que también está bien, vamos"

Entrevista 5. Joven usuaria de alcohol y drogas sintéticas.
Factores que influyen en el uso de preservativos, habiendo o no consumido drogas recreativas

La segunda línea discursiva encontrada agrupa aquellas opiniones que argumentan que las conductas sexuales que incrementan el riesgo de infección por VIH u otras ITS no tienen que ver con el consumo de drogas recreativas, o no más allá del hecho de que muchos encuentros sexuales se producen los fines de semana, tras salir de marcha y haber consumido alguna sustancia psicoactiva.

“¿qué más da que vaya el tío puesto o no puesto? Si en realidad es que no tiene condones en casa, no lo tiene metido en la cabeza, entonces que, (con drogas), lo único que hace es proyectar lo mismo."

Grupo 2. Varones y mujeres de 25 a 29 años consumidores de otras drogas además de alcohol y/o cannabis.

Los principales argumentos que emplean para explicar sus prácticas sexuales de riesgo son la falta de preservativos, la confianza en la pareja, la concepción del sexo como algo irracional e irrefrenable, el enamoramiento y el estado de ánimo o nivel de autoestima en el caso de las mujeres.

En primer lugar, la ausencia de preservativos aparece a menudo entre las razones por las que llevan a cabo prácticas sexuales de riesgo, habiendo o no consumido sustancias psicoactivas. La no disponibilidad de los mismos es justificada por su elevado precio, aunque en las y los adolescentes consultados también se observa falta de información sobre instituciones $u$ organizaciones que los distribuyen de manera gratuita. A las dificultades de acceso al preservativo añaden la imprevisión del encuentro sexual, especialmente las adolescentes y los jóvenes que no tienen pareja estable.

"y luego, ¿cuánto valen? Diez euros, janda hombre! Tanto 'usarlo, usarlo'. Pues dámelo, janda, hombre!"'

Grupo 3. Mujeres y varones de 18 a 24 años consumidores otras drogas además de alcohol y/o cannabis.

Por otro lado, los y las participantes consideran que la confianza en la pareja, así como la estabilidad de la misma, influye en una disminución de los riesgos sexuales percibidos, facilitando en relaciones homosexuales o heterosexuales que se practique la penetración sin preservativo, y en relaciones lésbicas prácticas, como la "tijera" (frotación vulba/vulba) en el periodo de menstruación, que incrementan el riesgo de infección por VIH. 


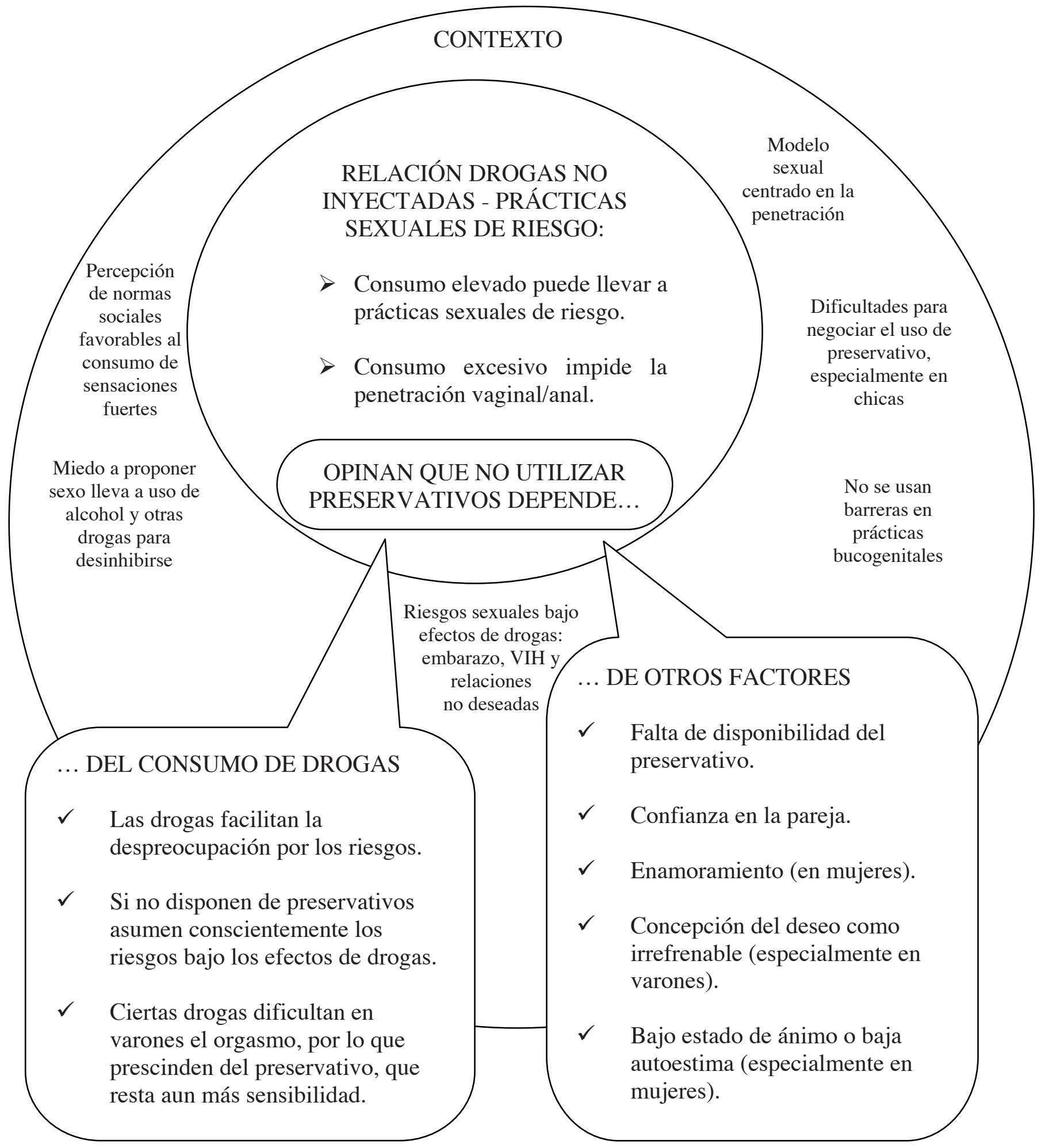

Figura 1. Principales opiniones de adolescentes y jóvenes participantes en la investigación 
"a mí me resulta difícil pensar que tengo confianza con una persona, si estoy aquí previniendo que me pegue algo (...) si no confío no follo y si confío, no uso condón"

Grupo 2. Varones y mujeres de 25 a 29 años consumidores de otras drogas además de alcohol y/o cannabis.

Un argumento para realizar prácticas sexuales de riesgo, especialmente recurrente entre las mujeres participantes, es el hecho de sentirse enamoradas, independientemente de la confianza o estabilidad que sientan con la pareja sexual en cuestión. Es una de las razones que consideran más influyentes para realizar prácticas sexuales de riesgo, independientemente de los distintos perfiles y hábitos de uso de drogas recreativas.

"Si tú estás muy enamorada, te condiciona más (...), ¿por qué crees que yo me preñé con 18 años?"

Grupo 7. Mujeres gitanas de 18 a 24 años consumidoras de alcohol y/o cannabis.

Asimismo, la concepción del deseo sexual como algo irrefrenable, donde se piensa que razonar resta placer, justifica la no utilización de preservativos en sus relaciones sexuales con penetración. Esta creencia aparece especialmente en las declaraciones de varones de todas las edades y en las de algunas mujeres menores de 18 años.

"El sexo es muy primario para controlarlo y decir -venga ponte-."

Grupo 12. Varones gitanos de 16 y 17 años consumidores de alcohol y/o cannabis.

Por último, el nivel bajo de autoestima o un bajo estado de ánimo se asocia, en opinión de algunas mujeres, a la pérdida de control en la negociación del preservativo. Los varones, sin embargo, creen que se trata más bien de falta de firmeza en la actitud, y declaran que con tal estado de ánimo no mantendrían relaciones sexuales.

"Que sí, hay mujeres a las que sí les afecta porque están ahí de bajón, pues como que se dejan llevar, ¿no?"

Grupo 4. Mujeres de 18 a 24 años consumidoras de alcohol y/o cannabis.

\section{Intervenciones preventivas}

De las campañas de prevención de drogodependencias las y los participantes valoran aquellas enmarcadas en la filosofía de la "reducción de riesgos o daños." En general, las y los jóvenes participantes consideran que los mensajes prohibicionistas deberían evitarse, así como informar más sobre las consecuencias a largo plazo del consumo de cada sustancia.

"a mí, porque me pongan un anuncio no me voy a dejar de drogar, pero por lo menos dime, -el speed descalcifica los huesos, itoma calcio niña!-."

Grupo 10. Mujeres de 18 a 24 años consumidoras otras drogas además de alcohol y/o cannabis.

Sugieren que se distribuya información en los lugares donde se consumen drogas, pero también en otros momentos más propicios para su lectura.

Las personas consultadas consideran que en su entorno ha disminuido el número de campañas e intervenciones sobre el VIH/SIDA. Entre las recomendaciones que plantean destaca la idea de que se evite dar en ellas una imagen negativa de la sexualidad.

"Yo creo que el tema es, continuar con la cultura del miedo, para mí ha llegado a significar eso (...) Porque el sexo antes era pecado mortal y ahora es peligro mortal"

Grupo 2. Varones y mujeres de 25 a 29 años consumidores de otras drogas además de alcohol y/o cannabis.

Las jóvenes lesbianas consultadas consideran que apenas existe información sobre la posibilidad de contagio en relaciones sexuales entre mujeres, lo que puede generar un sentimiento de invulnerabilidad en mujeres con esta orientación sexual.

"porque no, vamos, no hay estudios en el tema de las lesbianas (...) en el fondo, la verdad es que yo tengo la sensación de que las lesbianas no se contagian con eso"

Grupo 14. Mujeres lesbianas de 18 a 24 años consumidoras de alcohol y/o cannabis.

Por último, las personas gitanas menores de 18 años, participantes en la devolución de resultados y en el grupo nominal, sugieren para fomentar el uso de preservativos que estos se repartan gratuitamente en espacios públicos, como en la puerta de los centros educativos, en la calle o en bares. También señalan dar información en los centros educativos para concienciar a la gente desde pequeña, así como su difusión en la radio y discotecas.

Particularidades del colectivo gitano, como el escaso uso declarado de drogas -salvo tabaco y quizás psicofármacos- entre las mujeres consultadas, el valor de la virginidad en las solteras, el hecho de no ver necesario el uso de preservativos en las relaciones estables, o las confusiones sobre las formas de trans- 
misión del VIH sugieren intervenciones que tengan en cuenta lenguaje, creencias y prácticas comunes a estas poblaciones.

\section{DISCUSIÓN}

En este trabajo se han recogido una serie de dimensiones que afectan a las relaciones sexuales de adolescentes y jóvenes y a sus hábitos de consumo de drogas recreativas. Si se quiere profundizar en la influencia que los distintos usos de sustancias psicoactivas puede tener en la realización de prácticas sexuales de riesgo, es necesario tenerlas en cuenta. En general, los estudios cuantitativos tienen como objetivo dar una respuesta dicotómica a la cuestión sobre si el consumo de drogas facilita o no la realización de conductas sexuales de riesgo, lo que no permite la metodología empleada en este trabajo. Asimismo, algunos de los revisados presentan ciertas limitaciones en cuanto a la definición que utilizan de conductas sexuales de riesgo y de otras variables empleadas o ausentes ${ }^{28}$. Por ello se planteó, también como producto de esta investigación, construir un cuestionario mediante la triangulación de tres fuentes; los resultados de la investigación cualitativa, ítems e indicadores utilizados por los principales organismos nacionales e internacionales (ONUSIDA, OMS, etc.) y una revisión bibliográfica de indicadores utilizados en otros trabajos de investigación sobre la cuestión. El cuestionario fue pilotado con alumnado universitario de segundo año de la carrera de Psicología en Huelva (España). Las observaciones derivadas de este primer pilotaje permitieron mejorarlo, y hoy se encuentra a disposición de la comunidad científica.

Por otro lado, apenas se han localizado investigaciones cualitativas en revistas científicas sobre la temática de estudio. Entre ellas, trabajos como los de Morrison et al. ${ }^{36}$ y Fortenberry et al. ${ }^{37}$ no encuentran relación entre ambos comportamientos, mientras que los hallazgos de Friedman et al. apuntan a un mayor uso de preservativos en jóvenes que no tienen problemas con el alcohol $^{42}$.

Teniendo en cuenta que el $31 \%$ de los jóvenes españoles de 13 a 29 años declara que nunca consume alcohol, y un porcentaje mayor que no probaría distintas drogas ilegales ${ }^{43}$, se decidió entrevistar a un joven no consumidor de drogas, encontrándose en la triangulación redundancia entre su discurso y el de participantes usuarios habituales de drogas recreativas.

En la investigación de Calafat et al. ${ }^{44}$, el $45 \%$ de las y los jóvenes -la gran mayoría solteros- que consumen drogas ilegales y el $30 \%$ de los que solamente consumen drogas legales consideran que "buscar sexo" es una importante razón para salir de marcha. No obstante, es complejo determinar hasta qué punto las conductas sexuales de riesgo son producto del uso de drogas recreativas ${ }^{28}$, cuando se declara que éstas prácticas también se llevan a cabo sin estar bajo los efectos de sustancias psicoactivas ${ }^{45}$. O cuando declaran que el consumo abusivo de alcohol dificulta las relaciones sexuales con penetración, como referencian Bellis y Hughes ${ }^{46}$. Además, hay que tener en cuenta los diferentes efectos percibidos o esperados de cada sustancia. Por ejemplo, menores y jóvenes consultados no asocian el consumo elevado de cannabis con la realización de prácticas sexuales de riesgo, lo que parece estar en la línea de los hallazgos de Brodbeck et al. ${ }^{47}$, y no tanto en la del trabajo de Poulin y Graham ${ }^{48}$.

En definitiva, hay que tener en cuenta otros factores o motivadores que adolescentes y jóvenes consideran relacionados con la no utilización de preservativos. Por ejemplo, y en consonancia con los hallazgos de Guttmacher et al. ${ }^{49}$, los participantes de esta investigación dan importancia a la falta de disponibilidad de preservativos para explicar sus prácticas sexuales de riesgo, especialmente bajo los efectos de ciertas drogas. También se ha observado una percepción diferencial del riesgo en función del tipo de pareja, estable o casual, cuestión analizada en numerosos trabajos ${ }^{50,51,52}$. Las mujeres menores de 25 años hablaron de dificultades para negociar el uso de preservativos con algunos varones. Sus discursos son muy similares a los recogidos por Rodríguez San Julián, quien concluye que el sexo es "una práctica de riesgo cuando la razón es incompatible, y no puede competir, con el placer ni con el poder"53. Como en el trabajo de Holland et al. ${ }^{54}$, no parece ser solamente el comportamiento del varón el causante de estas asimetrías de poder, sino también las concepciones de las mujeres sobre la masculinidad y la feminidad. Destaca entre las jóvenes de la presente investigación el "enamoramiento" como factor de riesgo para no sentir la necesidad de utilizar preservativos ${ }^{55}$. Esto podría derivarse de una ideología de la feminidad que entiende el sexo como abandono del control en nombre del amor ${ }^{54}$.

Al igual que en otras investigaciones ${ }^{16,43,56}$, destaca en los varones consultados el discurso que relaciona el uso de preservativo con la pérdida de placer erótico. Esta disminución del placer, agravada bajo los efectos de ciertas drogas como la cocaína, que ellos igualmente asocian a la pérdida de sensibilidad sexual, les permite justificar el no uso del preservativo bajo los efectos de tal sustancia. Cuestión que podría explicar los resultados de McNall y Remafedi ${ }^{57}$.

Por último, mujeres relacionan la baja autoestima con la realización de prácticas sexuales de riesgo, relación con la que no se identifican los varones consul- 
tados. Los resultados de López y Moreno muestran ciertas similitudes, sin embargo, es otra la población de estudio y su trabajo no encuentra diferencias de género ${ }^{58}$.

Algunas recomendaciones que sugieren los resultados de esta investigación ${ }^{45}$ para intervenir en prevención de la transmisión sexual del VIH son las de publicitar una imagen erótica del preservativo y trabajar en la optimización del placer con jóvenes, para reducir la pérdida de sensibilidad percibida con el uso del condón. Igualmente, trabajar para normalizar el uso de preservativos y otras barreras en prácticas bucogenitales. Asimismo, de acuerdo con los hallazgos de Otegui ${ }^{59}$, convertir en objeto de campañas a las parejas estables, no sólo las relaciones casuales, y formar, especialmente a mujeres adolescentes, en habilidades sociales para negociar el uso del preservativo. Por último, mejorar la accesibilidad de los preservativos, especialmente en los espacios donde se consume alcohol y otras drogas.

Sintéticamente puede decirse que no existe unanimidad en las visiones que las personas consultadas en este estudio poseen sobre la influencia del consumo de drogas en las prácticas sexuales de riesgo de infección por VIH. Un consumo o policonsumo elevado de sustancias como alcohol o éxtasis se relaciona en ocasiones con una disminución en la preocupación por los riesgos asociados a una penetración sin preservativo. Si bien, señalan otros motivos para no utilizar preservativos, como el hecho de tener una pareja estable, donde los preservativos se sustituyen normalmente por otros métodos anticonceptivos. La no disponibilidad de preservativos, acompañada de una concepción del deseo como algo irrefrenable, o del enamoramiento en las mujeres parece llevar también a la realización de prácticas sexuales de riesgo, habiendo o no consumido drogas recreativas.

En definitiva, las cantidades consumidas de determinadas drogas, según las características personales de los sujetos, el tipo de relación que mantienen, y las actitudes previas, producto del contexto sociocultural y la educación sexual recibida, pueden influir en la realización de prácticas sexuales que incrementan el riesgo de infección por $\mathrm{VIH}$.

\section{AGRADECIMIENTOS}

Agradecemos a adolescentes y jóvenes su participación en la investigación; sin sus aportaciones desinteresadas este trabajo no hubiera sido posible. Igualmente queremos reconocer el importante apoyo financiero de la Consejería de Salud de la Junta de Andalucía y de la Fundació Barcelona SIDA 2002.

\section{REFERENCIAS}

1 WHO. Lexicon of alcohol and drug terms published by the World Health Organization. 2004. Recuperado el 25 de enero de 2005, de http://www.who.int/substance_ abuse/terminology/who_lexicon/en/.

2 E.M.C.D.D.A. Annual Report 2005. Recuperado el 22 de mayo de 2006, de http://www.emcdda.europa. eu/?nNodelD=419.

3 Calafat A, Montserrat J, Becoña E, Fernández C, Gil E, Palmer A, et al. Salir de marcha y consumo de drogas. Plan Nacional sobre Drogas. Recuperado el 6 de junio de 2006, de http://www.pnsd.msc.es/Categoria2/ publica/pdf/salir.pdf.

4 Durant RH, Smith JA, Kreiter SR, Krowchuck DP. The relationship between early age of onset of initial substance use and engaging in multiple health risk behaviours among young adolescents. Arch Pediatrics Adolescents Medicine 1999; 153: 286-291.

5 Settertobulte W, Bruun B, Hurrelmann K. Drinking among young europeans. WHO, Regional Office for Europe; 2001.

6 Grup Igia y colaboradores. Documento marco sobre reducción de daños. Gestionando las drogas. Conferencia de consenso sobre reducción de daños relacionados con las drogas: cooperación e interdisciplinariedad. Barcelona: GRUP IGIA; 2001.

7 Romo N. Mujeres y drogas de síntesis: género y riesgo en la cultura del baile. Donostia: Gakoa Liburnak; 2001.

8 Stiffman AR, Dore P, Cunningham RM, Earls F. Person and environment in HIV risk behavior change between adolescence and young adulthood. Health Educ Quart 1995; 22: 211-226.

9 Douglas, M. La aceptabilidad de riesgo según las ciencias sociales. Barcelona: Paidós; 1996 [original 1985].

10 Bimbela JL y Cruz MT. Sida y jóvenes: la prevención de la transmisión sexual del VIH. Granada: Escuela Andaluza de Salud Pública; 1997.

11 UNICEF. Hoja de datos del UNICEF: los jóvenes y el VIH/SIDA.1-2-2002. Recuperado el 25 de enero de 2005, de http://www.unaids.org/html/pub/topics/ youngpeople/unicefyoungfs_sp_pdf.pdf.

12 WHO, UNAIDS. Epidemic Update: December 2003. Recuperado el 5 de junio de 2006, de http://www.who. int/hiv/pub/epidemiology/epi2003/en/print.html.

13 UNAIDS. 2006 Report on the global AIDS epidemic. Recuperado el 5 de junio de 2006, de http://www. unaids.org/en/HIV_data/2006GlobalReport/default.asp.

14 UNAIDS, WHO. Situación de la epidemia de SIDA: 2005. Recuperado el 24 de marzo de 2006, de http:// www.unaids.org/epi/2005/doc/report_pdf.asp.

15 INJUVE. Informe de Juventud en España 2000. Recuperado el 5 de junio de 2006, de http://www. injuve. mtas.es/injuve/contenidos. item.action? id=6051 69259\&menuld $=1627100828$.

16 INE. Salud y hábitos sexuales. Las conductas sexuales desde la perspectiva del SIDA. Boletín Informativo del 
Instituto Nacional de Estadística 2004. Recuperado el 7 de junio de 2006, de http://www.ine.es/revistas/ cifraine/cifine_sida0704.pdf.

17 Rhodes T. Risk theory in epidemic times: sex, drugs and the social organization of "risk behaviour". Sociol Health IIIn 1997; 9: 208-227.

18 Ramrakha S, Caspi A, Dickson N, Moffitt TE, Paul C. Psychiatric disorders and risky sexual behaviour in young adulthood: cross sectional study in birth cohort. BMJ 2000; 321: 263-266.

19 Caballero HR, Villasenor SA. Socioeconomic strata as a predictor factor for constant condom use among adolescents. Rev. Saúde Pública. 2001; 35: 531-538.

20 Crosby RA, Hotgrave DR, DiClemente RJ, Wingood GM, Gayle JA. Social Capital as a Predictor of Adolescents' Sexual Risk Behavior: A State-Level Exploratory Study. AIDS and Behavior 2003; 7: 245-52.

21 Cáceres CF, Marin BV, Hudes ES, Reingold AL, Rosasco AM. Young people and the structure of sexual risks in Lima. AIDS 1997; 11: 567-577.

22 WHO, UNAIDS. The Rapid Assessment and response guide on Psycoactive Substance Use and Sexual Risk Behaviour. Recuperado el 19 de enero de 2004, de http://who.int/substance_abuse/publications/ epidemiology/en/-22k-.

23 Rodríguez A, Cabrera A, Jiménez JM, Hernán M. Opinions of adolescents and young spanish gypsies at risk of social exclusion about non-injectable drugs and sexual risks of hiv infection. Manuscrito remitido para publicación.

24 Fundación Secretariado General Gitano (FSGG), EDIS. Población Gitana y Empleo. Un estudio comparado. Madrid: FSGG; 2005.

25 Red Europea Sastipén. Documento Marco: La comunidad gitana europea y los fenómenos de las drogas y el VIH/SIDA. Recuperado el 20 de mayo de 2004, de www.fsgg.org/sastipen/S_doc2.htm.

26 Bezunartea P. La prevención del VIH/SIDA en la comunidad gitana. En: del Amo J, Belza MJ, Castillo S, Yacer A. Prevención del VIH/SIDA en inmigrantes y minorías étnicas, coordinadores. Madrid: Ministerio de Sanidad y Consumo; 2001. p. 96-99.

27 European Monitoring Centre on Racism and Xenophobia (EUMC). Breaking the Barriers - Romani Women and Access to Public Health Care. Luxembourg: Office for Official Publications of the European Communities; 2003.

28 Rodríguez A, Hernán M, Cabrera A, Romo N, García JM, Gutiérrez JL. ¿Tienen adolescentes y jóvenes que consumen drogas no inyectadas mayor probabilidad de transmisión sexual del VIH? Adicciones 2006; 18: 61-72.

29 Friedman SR, Flom PL, Kottiri BJ, Neaigus A, Sandoval $M$, Curtis $R$, et al. Consistent condom use in the heterosexual relationships of young adults who live in a high-HIV-risk neighbourhood and do not use "hard drugs". AIDS Care 2001; 13: 285-96.

30 Zablotska IB, Gray RH, Serwadda D, Nalugoda F, Kigozi G, Sewankambo N, et al. Alcohol use before sex and
HIV acquisition: a longitudinal study in Rakai, Uganda. AIDS 2006; 20: 1191-1196.

31 Liau A, DiClemente RJ, Wingood GM, Crosby RA, Williams KM, Harrington $\mathrm{K}$ et al. Associations between biologically confirmed marijuana use and laboratoryconfirmed sexually transmitted diseases among African American adolescent females. SexTransm Dis 2002; 29: 387-90.

32 McNall M, Remafedi G. Relationship of amphetamine and other substance use to unprotected intercourse among young men who have sex with men. Arch Pediatr Adolesc Med 1999; 153: 1130-5.

33 Duncan SC, Strycker LA, Duncan TE. Exploring associations in developmental trends of adolescent substance use and risky sexual behavior in a high-risk population. Behav Med 1999; 22: 21-34.

34 Apostolopoulos Y, Sonmez S, Yu CH. HIV-risk behaviours of American spring break vacationers: a case of situational disinhibition? Int J STD AIDS 2002; 13: 733-43.

35 Santelli JS, Robin L, Brener ND, Lowry R. Timing of alcohol and other drug use and sexual risk behaviors among unmarried adolescents and young adults. Perspect Sex Reprod Health 2001; 33: 200-5.

36 Morrison DM, Gillmore MR, Hoppe MJ, Gaylord J, Leigh BC, Rainey D. Adolescent drinking and sex: findings from a daily diary study. Perspect Sex Reprod Health 2003; 35: 162-8.

37 Fortenberry JD. Alcohol, drugs, and STD/HIV risk among adolescents. AIDS Patient Care STDS 1998; 12: 783-6.

38 Fromme K, D'Amico E, Katz E. Intoxicated Sexual Risk Taking: An Expectancy or Cognitive Impairment Explanation? J Stud Alcohol 1999; 60: 54-63.

39 Rhodes T, Stimson GV. What is the relationship between drug taking and sexual risk? Social relations and social research. Sociol Health IIIn 1994; 16: 219-28.

40 Linares N, Cravioto P. Principales enfoques y estrategias metodológicas empleados en la investigación del consumo de drogas: la experiencia en México. Rev Cubana Med Gen Integr [serial online] 2003 Mar-Apr; 19(2). Recuperado el 8 de junio de 2006, de http://www.bvs.sld.cu/revistas/mgi/vol19_2_ 03/mgi12203.htm

41 Atlas.ti. Version 5.0. Berlin: Scientific Software Development; 2003-2006.

42 Friedman SR, Flom PL, Kottiri BJ, Neaigus A, Sandoval $M$, Curtis $R$, et al. Consistent condom use in the heterosexual relationships of young adults who live in a high-HIV-risk neighbourhood and do not use "hard drugs". AIDS Care 2001; 13: 285-96.

43 Hernán M, Ramos M, Fernández. Salud y Juventud. Madrid: Consejo de la Juventud de España; 2002.

44 Calafat A, Fernández C, Juan M, Becoña E. Gestión de la vida recreativa: ¿Un factor de riesgo determinante en el uso reciente de drogas? Adicciones 2005; 17 (4): 337-47. 
45 Cabrera A, García JM, Gutiérrez JL, Hernán M, Rodríguez A, Romo N. Consumo de drogas no inyectadas y conductas sexuales de riesgo de infección por VIH en adolescentes y jóvenes. Granada: Escuela Andaluza de Salud Pública; 2004. Nº de expediente: 135/02. Financiado por la Consejería de Salud de la Junta de Andalucía y por la Fundació Barcelona SIDA 2002.

46 Bellis $M$ \& Hughes K. Pociones sexuales. Relación entre alcohol, drogas y sexo. Adicciones 2004; 16 (4): 249-58.

47 Brodbeck J, Matter M, Moggi F. Association Between Cannabis Use and Sexual Risk Behavior Among Young Heterosexual Adults. AIDS Behav 2006; 10 (5): 599605.

48 Poulin C \& Graham L. The association between substance use, unplanned sexual intercourse and other sexual behaviours among adolescent students. Addiction 2001; 96: 607-621.

49 Guttmacher S, Lieberman L, Ward D, Freudenberg N, Radosh A, Des Jarlais D. Condom availability in New York City public high schools: Relationships to condom use and sexual behavior. Am J Public Health 1997; 87: 1427-33.

50 Ferguson A, Pere M, Morris C, Ngugi E, Moses S. Sexual patterning and condom use among a group of HIV vulnerable men in Thika, Kenya. Sex Transm Infect 2004; 80: 435-9.

51 Bimbela JL. Juventud y sida: Análisis de conocimientos, actitudes y prácticas en Andalucía. [Tesis doctoral]. Barcelona: Universitat de Barcelona; 1999.
52 Lameiras M, Failde JM. Sexualidad y salud en jóvenes universitarios/as: actitudes, actividad sexual y percepción de riesgo de la transmisión heterosexual del VIH. Análisis y modificación de conducta 1997; 23: 27-63.

53 Rodríguez San Julián, E. Sexo y riesgo. La dialéctica entre el placer y la razón. Estudios de Juventud 2003; 63: 27-36.

54 Holland J, Ramazanoglu C, Scott S, Sharpe, Thomson R. Risk, power and the possibility of pleasure: young women and safer sex. AIDS Care 1992; 4: 273-83.

55 Romo N, Rodríguez A, Cabrera A, Hernán M. Girl Power: Recreational Drug Use, Gender Identity and Risky Sexual Behaviour among Spanish Youth. Manuscrito remitido para publicación.

56 López F, Moral J. Sexo, autoeficacia y actitud hacia las conductas sexuales de riesgo en la población adolescente. Medicina Universitaria 2003; 5 (18): 3-11.

57 McNall M, Remafedi G. Relationship of amphetamine and other substance use to unprotected intercourse among young men who have sex with men. Arch Pediatr Adolesc Med 1999; 153: 1130-5.

58 López MT, Moreno B. La depresión y la autoestima como predictores de comportamientos de alto riesgo para la infección de VIH (SIDA). Comunicación presentada al II Congreso Iberoamericano de Psicología. Madrid, 13-17 de julio de 1998.

59 Otegui Pascual, R. Investigación cualitativa sobre conocimientos, actitudes y comportamientos relacionados con la transmisión del VIH-SIDA en la población gitana. Madrid: II Convocatoria FIPSE; 2002. 
